INPLASY

PROTOCOL

To cite: Dai et al. Evaluation of the efficacy of medications for heart failure: a systematic review and network metaanalysis. Inplasy protocol 2020110004. doi:

10.37766/inplasy2020.11.0004

Received: 02 November 2020

Published: 02 November 2020

Corresponding author:

Hengheng Dai

csdy1114@sina.com

Author Affiliation:

Beijing University of Chinese Medicine

Support: The Fundamental Research Funds.

Review Stage at time of this submission: Preliminary searches.

Conflicts of interest:

None.

\section{Evaluation of the efficacy of medications for heart failure: a systematic review and network meta-analysis}

Dai, $\mathrm{H}^{1}$; Li, H²; Wang, B3; Wang, J4; Zhang, J5; Chen, Y6; Zhang, $\mathrm{X}^{7}$; Guan, Z8; Hu, M9; Liu, Y10; Shang, $\mathrm{H}^{11}$.

Review question / Objective: What are the effects of different drugs on all-cause mortality and readmission rates in patients with heart failure?

Condition being studied: We searched the databases of PubMed, Web of Science, EMBASE, and the Cochrane Controlled Trial registry from January 1, 1991 to October 6, 2020 using the following key words: heart failure AND All guideline-recommended drug classes: ACEls, BBs, ARBs, and MRAs and an ARNI, administered alone or in combination. There will be no restrictions imposed on the duration of studies, the type of allocation, the language or the publication period.

INPLASY registration number: This protocol was registered with the International Platform of Registered Systematic Review and Meta-Analysis Protocols (INPLASY) on 02 November 2020 and was last updated on 02 November 2020 (registration number INPLASY2020110004).

\section{INTRODUCTION}

Review question / Objective: What are the effects of different drugs on all-cause mortality and readmission rates in patients with heart failure?
Condition being studied: We searched the databases of PubMed, Web of Science, EMBASE, and the Cochrane Controlled Trial registry from January 1, 1991 to October 6, 2020 using the following key words: heart failure AND All guidelinerecommended drug classes: ACEls, BBs, 
ARBs, and MRAs and an ARNI, administered alone or in combination. There will be no restrictions imposed on the duration of studies, the type of allocation, the language or the publication period.

\section{METHODS}

Participant or population: Patients diagnosed with HF and aged over 18 years old but with no limitations on gender, course and comorbidity will be eligible.

Intervention: ACEIs/BBs/ARBs/ MRAs/ ARNI oral. Treatments can be associated with another therapy as long as a control group is present.

Comparator: The comparators will be placebo, and the different medications used to treat HFrEF : ACEls, BBs, ARBs, MRAs and so on.

Study designs to be included: All peerreviewed, full-reported prospective parallel randomised controlled trials (RCTs) irrespective of blinding will be assessed, but non-RCTs, small sample studies (less than 20 cases), duplicate reports and pilot studies will be excluded. In addition, the first phase data of randomised crossover trials and cluster RCTs will also be included for analysis.

Eligibility criteria: All peer-reviewed, fullreported prospective parallel randomised controlled trials (RCTs) irrespective of blinding will be assessed, but non-RCTs, small sample studies (less than 20 cases), duplicate reports and pilot studies will be excluded. In addition, the first phase data of randomised crossover trials and cluster RCTs will also be included for analysis.

Information sources: PubMed, Web of Science, EMBASE, and the Cochrane Controlled Trial registry.

Main outcome(s): Our primary outcomes will include all-cause mortality, HF-related death, all-cause rehospitalisation, clinical events and HF-related rehospitalisation.
Quality assessment / Risk of bias analysis: Risk of bias for each study was assessed by 2 of 3 independent reviewers for each outcome, separately. The Cochrane Collaboration revised tool was used to assess risk of bias in RCTs (RoB 2.0). Discrepancies were resolved by consensus. Based on the randomization process, deviation from expected interventions, lack of outcome data, outcome measurement and selection of the reported results, the trials were judged to have high risk of bias, low risk of bias or some concerns about bias. When all domains were considered to be low risk, the overall risk of bias was low. When at least one domain was high risk or at least three domains were considered to have some concerns, the overall risk of bias was high. A comparison-adjusted funnel plot was plotted to detect any dominant publishing bias in network meta-analysis. We evaluated the impacts of small-study for changes in LVEF of comparison adjusted funnel plot.

Strategy of data synthesis: First, pairwise meta-analyses with the fixed-effects model were conducted based on direct evidence using RevMan software (version 5.3.5). We evaluated the statistical heterogeneity of each pairwise comparison using the $I^{2}$ statistic and $p$ value. Treatment effects were estimated as standardised mean difference (SMD) for continuous outcomes with 95\% Cls. Second, a frequent random-effects network meta-analysis was performed with a multivariate meta-analysis model, using the mumeta commands and routines in Stata, in order to synthesize the results of multiple interventions of direct and indirect evidence. The results of network metaanalysis were summarised with effect sizes (SMD) and credible intervals (CrI). We constructed a network diagram of the outcome, with the node size represented the number of patients randomly assigned to each intervention, and the line thickness between nodes corresponded to the number of studies assessing each comparison. A sensitivity analysis of the result was conducted, including only trials at low risk of bias. 
Subgroup analysis: In the network of primary outcome analysis classified by drug species, patients were allocated to 5 interventions: ACEIs, BBs, ARBs, MRAs, and ARNI.

Sensibility analysis: When the results of the data are highly heterogeneous, we will use the method of exclusion one by one for sensitivity analysis.

Language: English.

Country(ies) involved: China.

Keywords: Heart failure, Curative effect comparison, Meta-analysis of the network.

Contributions of each author:

Author 1 - Hengheng Dai - The author drafted the manuscript.

Author 2 - Haisong $\mathrm{Li}$ - The author provided statistical expertise.

Author 3 - Bin Wang - The author contributed to the development of the selection criteria, and the risk of bias assessment strategy.

Author 4 - Jisheng Wang - The author read, provided feedback and approved the final manuscript.

Author 5 - Jingjing Zhang - The author provided statistical expertise.

Author 6 - Ying Chen - The author contributed to the development of the selection criteria, and the risk of bias assessment strategy.

Author 7 - Xuecheng Zhang - The author contributed to the development of the selection criteria, and the risk of bias assessment strategy.

Author 8 - Zhiyue Guan - The author provided statistical expertise.

Author 9 - Mingzhi Hu - The author provided statistical expertise.

Author 10 - Yan Liu - The author read, provided feedback the final manuscript.

Author 11 - Hongcai Shang - The author read, provided feedback and approved the final manuscript. 